\title{
Research on the emotional cognitive evaluation model based on artificial neural network
}

\author{
Qiying Gan \\ School of Educational Science, Wenshan University, Wenshan 663000, Yunnan, China
}

\begin{abstract}
. the neural network, fuzzy set theory and evolutionary algorithm in artificial intelligence are all intelligent information processing theories that follow the biological processing mode. These theories are realized by rational logical thinking mode without considering the role of human perceptual thinking in the information processing process, such as emotion and cognition. Among them, the neural network mainly imitates the function of the mental system of human, adopts the method from the bottom to the top, and processes the difficult language pattern information through a large number of complicated connections of neurons. Artificial neural network (Ann) is a cross research field of artificial intelligence and life science. This theory mainly imitates the information processing mechanism of organisms in nature and is mainly used in intelligent information processing systems that can adapt to long-term changes in the environment. Therefore, neural network has important application significance in the research of intelligence, robot and artificial emotion.
\end{abstract}

\section{Artificial neuron model}

Artificial neuron model is based on the basic conditions for the creatures in the nature of information, with the need to connect neurons, as in the nature of biological information processing mechanism and connections between neurons network composition complex, its essence is through the simple artificial neuron densely connected guarantee have a certain number of real-valued output neurons, so as to realize input neurons units[1]. Artificial neural network is mainly imitate nature of biological information processing mechanism, as a result of biological information processing system is made up of interconnected neurons of the complex network, and therefore similar to biological via system, artificial neural network is made up of a series of simple artificial neuron densely connected each other, each of which has a certain number of artificial neurons real value input, the use of artificial neural network using the mathematical logic and the mathematical model to simulate some structure and function of the biological nervous system, including artificial neuron model as shown[2]. In figure 1, the inputoutput relationship of the artificial neuron model is

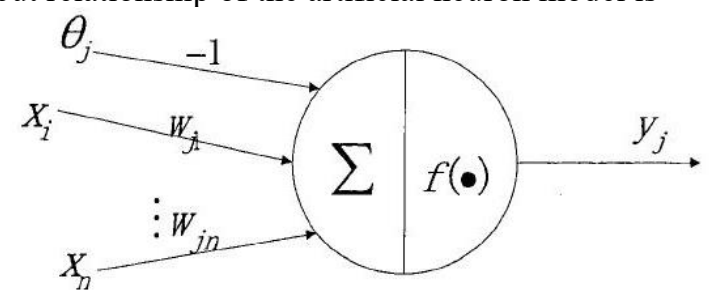

Fig. 1 Neuron model
The input and output relationship of the artificial neuron model is as follows:

$$
\begin{gathered}
S_{J}=\sum_{\mathrm{i}=1}^{n} \omega_{j i} x_{i}-\theta_{j}=\sum_{i=0}^{n} \omega_{j i} x_{i}\left(x_{o}=\theta_{j}, \omega_{j o}=-1\right) \\
\mathrm{y}_{i}=f\left(S_{j}\right)
\end{gathered}
$$

\subsection{BP network}

BP network is artificial neural network in the best simulation mechanism of biological treatment information network status, is composed of parallel nonlinear distributed information processing network rhythm, by most of the artificial neuron network connections between, everyone keep single bit output neurons, and each neuron can be connected to each other, can have connection weights connection pathways for output interface[3]. It mainly simulates the mechanism of biological processing information from two aspects: one is to simulate the biological neural structure and realize the biological mechanism, which involves many basic disciplines such as biology, physiology and cognitive neuroscience. However, the current science and technology is far from fully understanding the structure and mechanism of biological neural system. The other is to simulate some functions of the biological nervous system[4], that is, to make the artificial neural network with some functional characteristics of the biological nervous system, such as learning, decision-making and other functions. The latter is now being pursued by a wide range of ai researchers. According to the topology of the neural network, BP artificial neural network is divided into feedforward neural 
network and feedback channel network, as shown in figure 2.

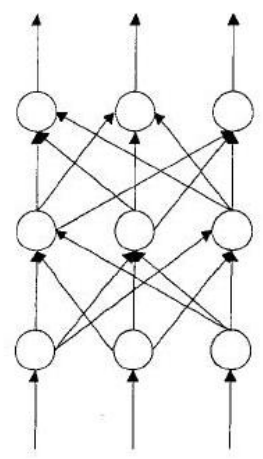

Fig 2 Front-end feedback network

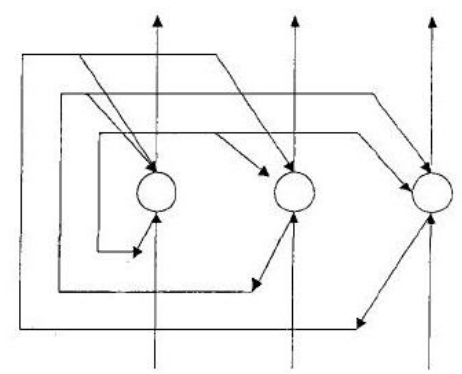

Fig. 3 feedback neural network

\subsection{Algorithm demonstration}

The specific steps (3) to implement the neural network algorithm are as follows: the initial weight coefficient is set to a random non-zero value with a small interval[5].

According to the given pairs of input and output samples, calculate the output of the network: assume that the input and output samples in group I are (4).

$$
\begin{gathered}
u_{p}=\left(u_{1 p}, u_{2 p}, \ldots \ldots u_{n p}\right) \\
D_{\mathrm{p}}=\left(d_{1 p}, d_{2 p}, \ldots d_{k p}\right) p=1.2 \ldots . L
\end{gathered}
$$

Group P samples are input at the node and the output is:

$$
\mathrm{y}_{i p}(t)=f\left[x_{i p}(t)\right]=f\left[\sum_{j} \omega_{i j}(t) I_{j p}\right]
$$

In order to take the asymmetric function, the function can be expressed by mathematical formula as follows:

$$
f(x)=\frac{1}{1+e^{-x}}
$$

The entire algorithm flow chart is shown in figure 4 :

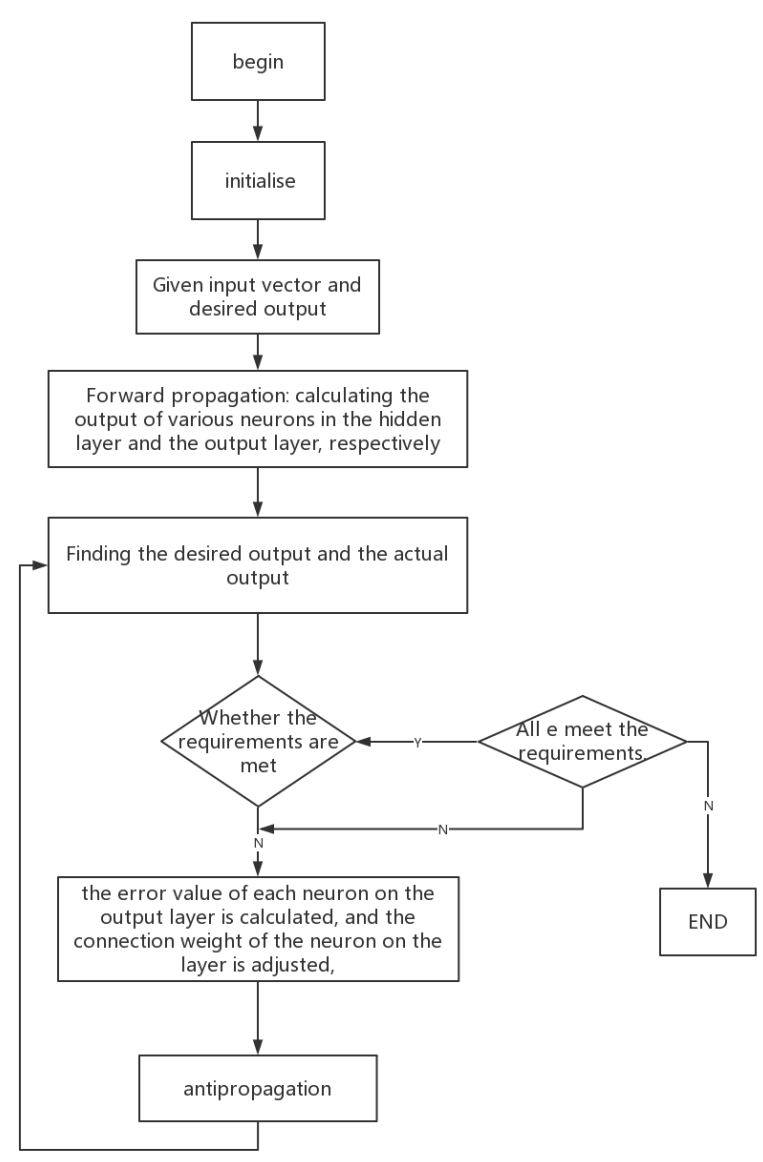

Fig 4 flow chart of BP algorithm

\section{Affective cognitive evaluation model}

\section{1 affective cognitive evaluation process}

Emotional ability is one of the emotional theories proposed by the American psychologist Arnold, which is the only difference between the current artificial intelligence. It refers to the ability to explore cognitionrelated calculations in computers and artificial intelligence and to calculate relevant emotional factors triggered by sensibility [5]. To compare the speed at which humans can make better decisions and use knowledge. The acquisition of this ability is directly related to emotion, and the discipline of psychology, the discipline of cognitive science, the interpretation of emotion theory and physiological and psychological processes, and the analysis can show the difference between human beings and other intelligences. Emotional ability is one of the differences between human beings and current intelligent machines. Emotional computing refers to the calculation of factors related to emotions, triggered by emotions or able to affect emotions in a computer or machine. With the development of artificial intelligence to this day, human beings are far worse than computers in memory and calculation speed, but they have better ability to use knowledge to think and make decisions, which is largely due to the involvement of emotion. Researchers in 
psychology[6], cognitive science, and physiology attempt to systematically explain the physiological and psychological processes of emotions and their relationships through the study of emotion theory, nerves, and physiology. Understanding of the emotional cognitive theory, psychologists from different sides, such as emotional behavior, emotions physiological, cognitive, emotional expression and emotion recognition study put forward a lot of different points of view, and on the basis of them formed a variety of emotional cognitive theory, thus the produce process of emotional made many explanations. The physiological and psychological processes of emotion production are carried out simultaneously in the emotional experience, and any evaluation of things or events has the color of emotion. The evaluation of things or events by emotion is regarded as a corresponding activity tendency generated by perception. When such activity tendency is strong[7], it is called emotion. The emotional change caused by the evaluation of situational events will lead people to choose emotional behaviors suitable for the situation. Among them, since any evaluation of new things is based on past emotional experience, memory is regarded as the basis of evaluation. As shown in figure 5, since the current situation and related emotional memory will make us imagine its influence before the start of emotional behavior, imagination is considered as an important part of evaluation, and the whole complex evaluation process takes place almost instantaneously [8].

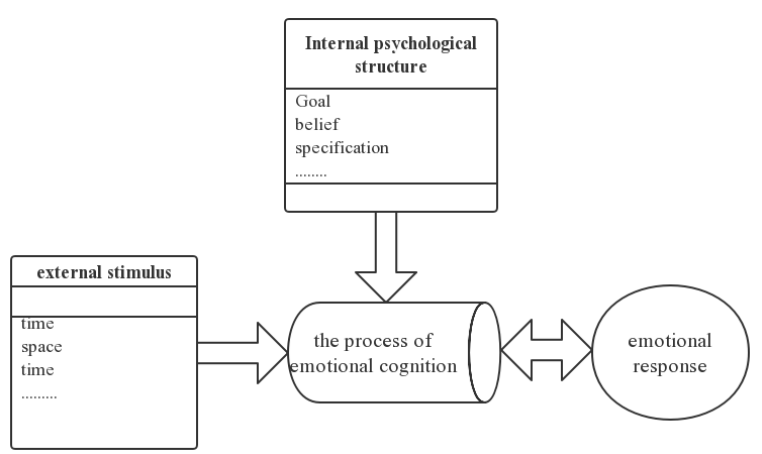

Fig 5. The process of emotion recognition and evaluation

\subsection{Affective cognitive evaluation model}

Such as cognitive psychology and artificial intelligence researchers pay more and more attention to in the field of emotion and cognition in People's Daily life and work can not be ignored in the role of the cognitive evaluation theory is that there exists a series of evaluation criteria used to distinguish different emotional state, so the different evaluation criteria will have different basic emotion classification methods and results[9]. Human emotion for emotion and cognition gradually realized that the psychology, and artificial intelligence can effectively refine research on the field, including emotional cognitive evaluation theory as an interdisciplinary theory of derivative products, and can take on a variety of representative theory, can carry on the mathematical quantification to emotional cognitive evaluation theory, the theory and application and computer can differentiate between a series of evaluation standards, and achieve different state, so the different emotional categories under different standards, the results are different, as shown in the figure 6 , the emotional evaluation of table structure[10].

Table.1 emotional evaluation structure table

\begin{tabular}{|c|c|c|c|c|c|c|}
\hline & unexpected & $\begin{array}{l}\text { Motivation } \\
\text { and context }\end{array}$ & probability & control bit & cause & problem types \\
\hline wonder & unexpected & 一 & 一 & 一 & 一 & - \\
\hline glad & 一 & promote & unanimous & & 一 & 一 \\
\hline Be relieved & 一 & & confirm & & & 一 \\
\hline sad & 一 & & & & & 一 \\
\hline constrain & 一 & 一 & promote & & 一 & 一 \\
\hline hope & 一 & 一 & atypism & Low control & & 一 \\
\hline fear & 一 & 一 & 一 & unanimous & 一 & 一 \\
\hline like & 一 & 一 & unanimous & 一 & 一 & 一 \\
\hline Dislike & 一 & atypism & 一 & Low control & 一 & 一 \\
\hline arrogant & 一 & & & & 一 & \\
\hline dispirited & 一 & 一 & Other reasons & 一 & 一 & Non-character \\
\hline
\end{tabular}


According to the simulation of the rhythm table of emotional evaluation, it can be concluded that when set in any time window, the affective cognitive decision is in an external observable state, but in a state, so the affective cognitive decision can be reflected in the model [11-12].

$$
\operatorname{Pr}\left(c^{\prime} \mid c, d\right)
$$

The emotional model is as follows:

$$
\operatorname{Pr}\left(e^{\prime} \mid c^{\prime}, e\right)
$$

The emotional transformation model is

$$
\begin{aligned}
\operatorname{Pr}\left(e^{\prime} \mid e_{p}, c, d\right) & =\sum_{i=1}^{|C|} \operatorname{Pr}\left(e^{\prime} \mid c^{\prime}=i, e_{p}\right) \operatorname{Pr}\left(c^{\prime}=i \mid c, d\right) \\
& =\sum_{i=1}^{|C|} \sum_{K=1}^{|E|} e_{p k} \operatorname{Pr}\left(e^{\prime} \mid c^{\prime}=i, e=k\right) \operatorname{Pr}\left(c^{\prime}=i \mid c, d\right)
\end{aligned}
$$

Affective cognitive decision-making model:

$$
\begin{gathered}
Q_{D M}(c, a, d)=Q_{e x t}(c, d)+Q_{\mathrm{mt}}(c, a, d) \\
\operatorname{Pr}(d=k \mid c, a)=\frac{\exp \left(\beta Q_{D M}(c, a, d)\right)}{\sum_{l=1}^{|D|} \exp \left(\beta Q_{D M}(c, a, l)\right)}
\end{gathered}
$$

\section{Experimental results and data analysis}

\section{1 experimental analysis}

In the experimental results, the data analyst will verify the algorithm in the visual environment, as shown in figure 7. According to the moving route and path of the agent at a specific target, it can be directly observed in the room. As a reference object in the detection, its agent can avoid obstacles in the path. When the agent perceives through observation, the external environment can conduct strategic avoidance and execute corresponding actions according to techniques, functions and other methods. In the process of movement, when the number of successes exceeds the number of failures, it is necessary to encourage and encourage. If obstacles cannot be successfully avoided, it is necessary to make timely allocation decisions and carry out action planning again.

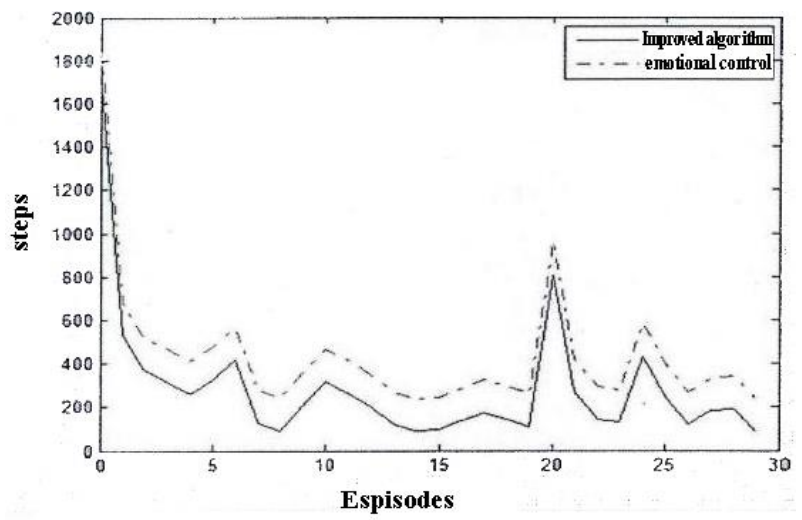

Fig. 6 Experimental analysis

\section{2 fitting index}

To measure the acceptability of a conceptual model, we mainly look at the fitting of the conceptual model and the observed data.

The main fitting indexes of this model are shown in table 8 below

Table 2 Best Fit Data

\begin{tabular}{cccccccccc}
\hline $\mathrm{X}^{2}$ & $\mathrm{df}$ & $\mathrm{x}^{2} / \mathrm{df}$ & rmsea & gfi & nfi & cfi & ifi & pnfi & pgfi \\
\hline 225.317 & 63 & 3.57 & 0.076 & 0.931 & 0.949 & 0.962 & 0.963 & 0.657 & 0.558 \\
\hline
\end{tabular}

\section{Conclusion}

the artificial neuron model in artificial neural network is discussed in this paper, based on the structure and basic principle of the divine network, this paper explores the affective cognitive process and affective cognitive evaluation theory by using neural network algorithm and specific implementation steps, and then summarizes the specific strategies of affective cognitive decision-making methods through affective cognitive evaluation theory.

\section{References}

1. Wei Wei,Jia Qingxuan. Weighted Feature Gaussian Kernel SVM for Emotion Recognition.[J]. Computational intelligence and neuroscience,2016,2016.

2. Matallana Diana,Gómez-Restrepo Carlos,Ramirez Paulina,Martínez Nathalie Tamayo,Rondon Martin.
[The Recognition of Emotions, Empathy and Moral Judgment in the National Mental Health Survey in Colombia, 2015].[J]. Revista colombiana de psiquiatria,2016,45 Suppl 1.

3. Brown Laura S. The Influence of Music on Facial Emotion Recognition in Children with Autism Spectrum Disorder and Neurotypical Children.[J]. Journal of music therapy,2016.

4. Luo Xin. Talker variability effects on vocal emotion recognition in acoustic and simulated electric hearing.[J]. The Journal of the Acoustical Society of America,2016,140(6).

5. Fridenson-Hayo Shimrit,Berggren Steve,Lassalle Amandine, Tal Shahar,Pigat Delia,Bölte Sven,BaronCohen Simon,Golan Ofer. Basic and complex emotion recognition in children with autism: crosscultural findings.[J]. Molecular autism,2016,7.

6. Tang Xiao Wei,Yu Miao,Duan Wei Wei,Zhang Xiang Rong,Sha Wei Wei,Wang Xiang,Zhang Xiao 
Bin. Facial emotion recognition and alexithymia in Chinese male patients with deficit schizophrenia.[J]. Psychiatry research,2016,246.

7. Dmitry Lyusin,Victoria Ovsyannikova. Measuring two aspects of emotion recognition ability: Accuracy vs. sensitivity[J]. Learning and Individual Differences,2016,52.

8. L. Gamond,Z. Cattaneo. The dorsomedial prefrontal cortex plays a causal role in mediating in-group advantage in emotion recognition: A TMS study[J]. Neuropsychologia,2016,93.

9. Dominic A. Trevisan,Elina Birmingham. Are emotion recognition abilities related to everyday social functioning in ASD? A meta-analysis[J]. Research in Autism Spectrum Disorders,2016,32.

10. Alini Daniéli Viana Sabino,Marcos Hortes N. Chagas,Flávia L. Osório. Effects of psychotropic drugs used in the treatment of anxiety disorders on the recognition of facial expressions of emotion: Critical analysis of literature[J]. Neuroscience and Biobehavioral Reviews,2016,71.

11. Yan Sun, Siyu Bo, Jiaojiao Lu . Brain network analysis of cognitive reassessment and expression inhibition of emotion regulation strategies: evidence from EEG and ERP [J/OL]. Journal of Psychology: 1 1411.1911.b.20191120.0935.010.html.

12. Jianzhong Wang, Luxue Gong, Junqing Song. The connotation type, psychological mechanism and adjustment intervention strategy of psychological pain: a new way to improve individual subjective well-being from the perspective of cognitive reassessment $[\mathrm{J}]$. Journal of Southwest University for nationalities (Humanities and Social Sciences Edition), 2019, 40 (10): 210 - 216. 